\title{
Analysis of Job Satisfaction based on Oracle Database
}

\author{
Wang Tao \\ Eastern Liaoning University, Dandong 118000, China
}

Keywords: Oracle database, Clustering, Fuzzy evaluation, C language, Dynamic data

\begin{abstract}
In order to evaluate the massive data sample, this paper uses clustering and fuzzy comprehensive evaluation theory to design the job satisfaction Oracle database. In order to increase easy operation of database, this paper uses $C$ language called form to establish the database interface, which realizes the dynamic operation of database data. This evaluation system can real-time extend sample data of job satisfaction, realizing the topology function of sample size and the quantity, which improves the analysis efficiency of sample data. Through calculation, the average level of job satisfaction is obtained, which provides a theoretical basis for the research on the employment of university students.
\end{abstract}

\section{Introduction}

Oracle database system is using the distributed database as the core developed by American Oracle, which is one of the most popular client server or B/S system structure. Oracle database is currently the most widely used in the world, as a general-purpose database system, it has complete data management capabilities; as a relational database, it is a complete relation product; as a distributed database, it implements distributed processing function [1, 2]. Using many advantages of Oracle, this paper designs dynamic assessment system of college students' job satisfaction, which achieves high-speed processing of massive data sample.

\section{The Overall Design of Job Satisfaction Oracle Database}

In order to make the job satisfaction investigation more close to the real results, this paper uses the written and erased functions of Oracle database to topology on sample. Through the test of different samples, the dynamic evaluation of satisfaction is achieved. The overall design of the system is shown in Figure 1.

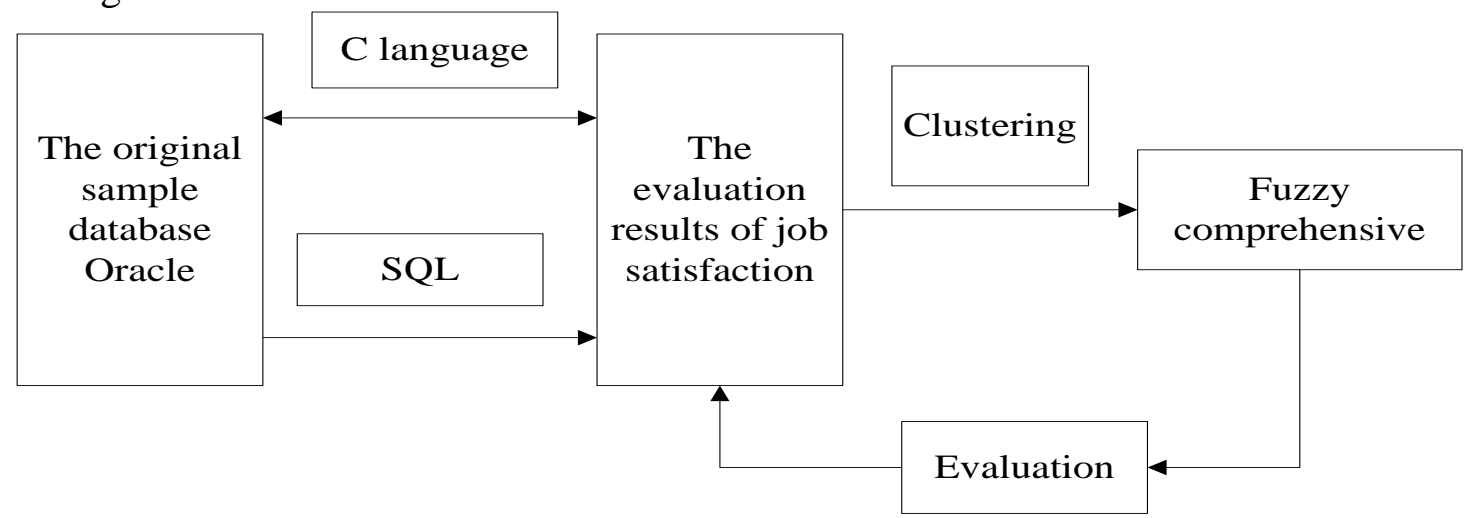

Fig. 1 Schematic diagram of employment satisfaction evaluation system

Figure 1 shows the overall design process of job satisfaction evaluation system. First it uses Oracle to establish job satisfaction database, and design cluster and comprehensive fuzzy evaluation algorithm to operate on the sample data, and uses C procedure call to realize data SQL transformation [3]. When the sample size and the number increase, it can edit and modify the database, and write new data dynamically, which realize the dynamic evaluation of job satisfaction and improves the accuracy of evaluation. 
The influence factors of job satisfaction have many aspects, and it can't get the desired effect evaluating the satisfaction from a single hand [4-6]. This paper adopts the comprehensive fuzzy evaluation method, and the weight coefficient of main influence factors is written $P=\left\{P_{1}, P_{2}, \cdots, P_{n}\right\}$, so the evaluation matrix can be obtained:

$$
Y=\left[\begin{array}{cccc}
p_{11} & p_{12} & \cdots & p_{1 n} \\
p_{21} & p_{22} & \cdots & p_{2 n} \\
\vdots & \vdots & & \vdots \\
p_{n 1} & p_{n 2} & \cdots & p_{n n}
\end{array}\right] .
$$

The $p_{i i}$ of $Y$ need to be known the number, assuming that the number of evaluation is $N$, so after the unified planning it can obtain:.

$$
\left\{\frac{T_{i 1}}{N}, \frac{T_{i 2}}{N}, \ldots, \frac{T_{i m}}{N}\right\} \text { and } \sum_{j=1}^{m} T_{i j}=N .
$$

Doing classification level evaluation, so judgment matrix is:

$$
\sum_{i=1}^{N} \frac{T_{i j}}{n}\left[e_{i}, f_{i}\right] \text {. }
$$

In order to realize the employment satisfaction assessment for different investigation objects, this paper uses clustering algorithm to do clustering on the evaluation interval. The expression of clustering function is:

$$
Y\left(y_{j}\right)=0.01+\sum_{i=1}^{n} \frac{1}{i+\sum_{j=1}^{n}\left(y_{j}-a_{j i}\right)^{n}} .
$$

Assuming the students samples of participation in employment satisfaction survey are $c_{k}=\left(c_{k 1}, c_{k 2}, \cdots, c_{k n}\right)$, so the clustering center of $c_{k}$ and $c_{l}$ is calculated as follows:

$$
\rho\left(c_{k}, c_{l}\right)=\sqrt{\sum_{z=1}^{b}\left(c_{k z}-c_{l z}\right)}, k, l=1,2, \cdots, n .
$$

The density of data sample point $b_{k}$ can be expressed as:

$$
M_{k}=\sum_{l=1}^{n} \frac{\rho\left(b_{k}, b_{l}\right)}{\sum_{k=1}^{n} \rho\left(b_{l}, b_{e}\right)}, k=1,2, \cdots, n .
$$

In order to achieve comprehensive evaluation of data samples and obtain the average level of employment, this paper uses Oracle database to manage and operate data. In order to increase easy operation, this paper uses $C$ language to design database interface to realize the operation of the database. And the main program is as follows:

public partial class Frm_manage: Form

\{

private DataRow myDataRow;

private DataTable myDataTable;

public static OracleConnection oracle_conn;

OracleCommand cmd;

OracleDataAdapter da;

public static string conn_string = "Data

Source=MYDB/MYPWD;Persist Security Info=True;User

ID=TRY;Password=TRY;Unicode=True";

public DataSet ds = new DataSet();

public Frm_manage(Form frm) 


\section{Design of Job Satisfaction Evaluation System based on Oracle Database}

In order to verify the effectiveness and reliability of integrated fuzzy clustering evaluation algorithm designed in the second section, this paper selects mass sample of job satisfaction to establish the Oracle database, and uses $C$ language calling form to operate the database, which realizes the comprehensive fuzzy evaluation of employment satisfaction.

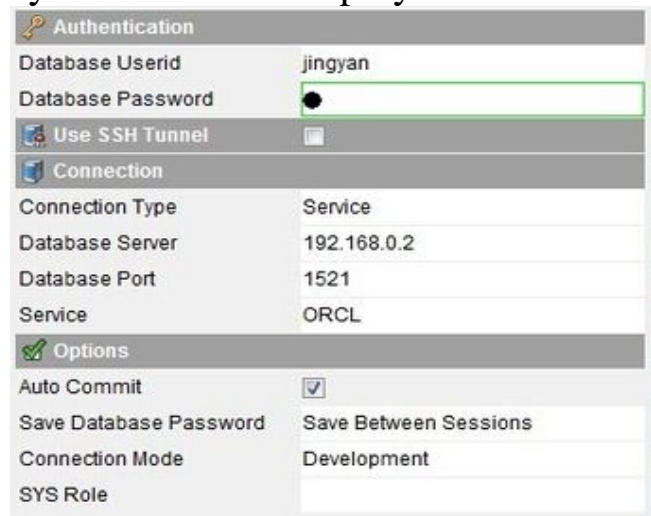

Fig. 2 The Oracle database connection

Figure 2 shows the database user operation terminal. It is composed of applications, tools, SQL* NET. When the user operates the database, it must be connected to a server, and the database is referred to as the local database [7]. Under the environment of network, DB on other servers is known as a remote database. If the user wants to access remote data on the $\mathrm{DB}$, it must establish a database link.

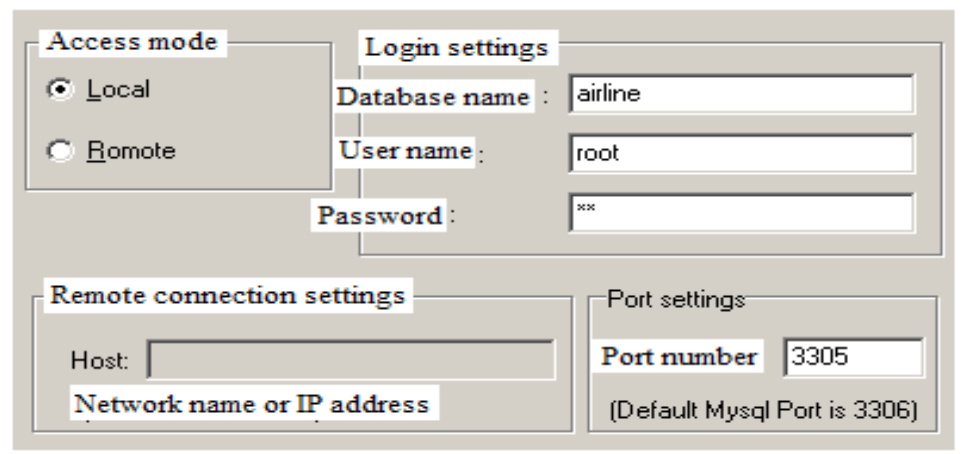

Fig. 3 C language database calling

In order to develop and edit the data samples as shown in Figure 3, it uses $C$ language calling form to convert the Oracle database to SQL [8]. The converted data computation speed is relatively stable, and the database has real-time write and delete functions.

\begin{tabular}{|c|c|c|c|c|}
\hline SQL & Elapsed Time & Response Time & Oracle Cost & Expert Rank \\
\hline Source & $00: 00: 55.86$ & $00: 00: 55.62$ & 13 & 68 \\
\hline SQL1 & $00: 00: 58.06$ & $00: 00: 57.95$ & 13 & 68 \\
\hline SQL2 & $00: 00: 55.66$ & $00: 00: 55.55$ & 13 & 68 \\
\hline SQL3 & & & 13 & 68 \\
\hline SQL4 & & & 13 & 68 \\
\hline SQL5 & & & 13 & 68 \\
\hline SQL6 & & & 13 & 68 \\
\hline SQL7 & & & 13 & 68 \\
\hline SQL8 & & 13 & 68 \\
\hline
\end{tabular}

Fig. 4 Database computing time

Figure 4 shows the calculation process of SQL. Through satisfaction investigation for 2600 samples in different jobs, it just costs 55 seconds. When executing the SQL command, it has two conditions: returning a scalar value and not returning a scalar value [9]. Using C language calling form can realize the database dynamically adding data function. 


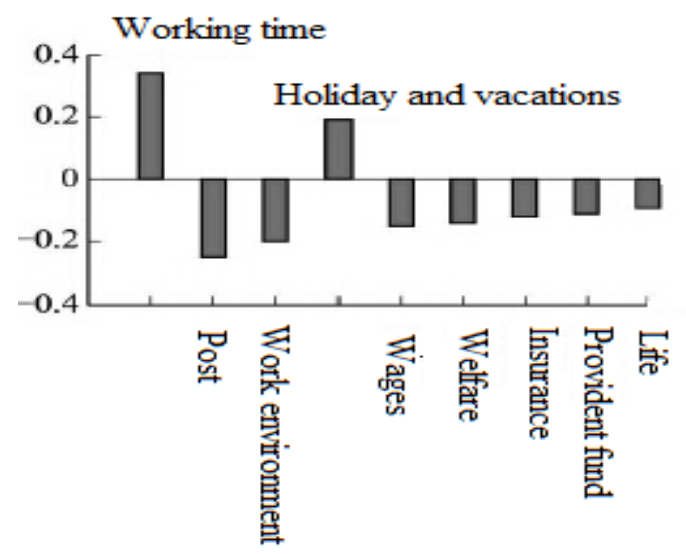

Fig. 5 The evaluation results of job satisfaction

Figure 5 shows the calculation results when sample number and capacity are expanded a fold [10]. From the chart it can be seen, in job satisfaction evaluation, in addition to the work time and holidays, the average level of satisfaction is low, and it is the same as the results before sample topology, which verifies the reliability of evaluation system.

\section{Summary}

Based on fuzzy comprehensive evaluation and clustering algorithm, this paper designs evaluation mathematics model of job satisfaction and establishes the Oracle database, and uses C language call form to realize the data SQL conversion. Through the operation and test of the database, the system can achieve real-time extension of job satisfaction sample size and quantity, which greatly improves the analysis efficiency and the accuracy of sample data. It is a new computer satisfaction analysis system.

\section{References}

[1] Z.F. Li, J. Song, B.Q. Liu, M. Wu, Z.H. Cao. Application of Oracle Forms in cold two levels HMI system. Chinese software, 2014, 2(7): 110-114.

[2] D.Z. Zhang, Z.F. Su, Y. Lin, Y.S. Xue. The top-k keyword query based on relational database. Journal of computer research and development, 2014, 4(4): 24-26.

[3] J.H. Gong. Data table end mobile sort algorithm based on data extraction. Office automation, 2014, 5(10): 45-48.

[4] T.T. Hu, M. Cao. Relational database storage model based on ontology theory. Computer engineering and design, 2014, 3(9): 56-59.

[5] J.Y. Liu. Research on OLAP based on relational database. Coal technology, 2013, 5(4): 78-82.

[6] F.P. Guo. Investigation analysis of university libraries characteristic database construction in Beijing. Science and technology innovation herald, 2012, 2(34): 92-95.

[7] H. Zhou, C. Zhang. Principle of library characteristic database construction. Old area building, 2013, 4(14): 32-36.

[8] H. Jiang. Study on college library featured database construction of in higher vocational. Science and technology innovation herald, 2012, 2(3): 45-48.

[9] S.J. Liu. Construction of Chengde tourism resource characteristic database. Journal of Hebei tourism of Career Academy, 2012, 5(3): 45-48.

[10]H. Xia, W. Zhang. The library characteristic database construction in military academies. Science and technology innovation herald, 2012, 3(21): 102-105. 